\title{
La acción tutorial: Análisis de la dinámica intelectual desde la praxis universitaria
}

\section{The tutorial action: Analysis of the intellectual dynamics from the university praxis}

Barrios B., Nanci C.

Jenny Andrea Quiroz Villacís

Universidad de Especialidades Espíritu Santo, Ecuador

Autor para correspondencia: nancbarrios@uees.edu.ec; jaquiroz@uees.edu.ec

Fecha de recepción: 23 de agosto de 2018 - Fecha de aceptación: 01 octubre de 2018

Resumen: Los estudios de post grado se han venido incrementando en los últimos años, tanto en cantidad ofertada como en la cantidad de participantes inscritos en los mismos, sin embargo el número de egresados no se corresponde con el número de inscritos, muchos culminan su carga académica más no cumplen con la presentación del Trabajo de titulación requisito para la obtención de la titularidad, por lo que la producción intelectual sigue sin responder las expectativas que el mercado y la sociedad requieren. Hasta ahora las diferentes investigaciones al respecto tratan de enfocar el problema hacia áreas muy específicas, sin embargo los procesos de pensamiento o la dinámica intelectual que entra en juego no parecieran haber despertado inquietudes para su investigación, de allí que la presente investigación pretendió identificar aquellos procesos que están implícitos durante el desarrollo de la praxis tutorial, para ello, la misma se enmarcó dentro de un enfoque mixto, donde lo cuantitativo se enmarcó en una investigación de tipo descriptivo, con diseño no experimental, la población estuvo conformada por 18 docentes con función de tutor, la información se obtuvo a través de una encuesta tipo Lickert, el enfoque cualitativo permitió a las investigadoras un acercamiento directo con su objeto de estudio que es la Dinámica Intelectual, la información se obtuvo por medio de entrevistas a informantes clave. El uso de diferentes estrategias: resúmenes, subrayado, cuestionamientos, entre otros quedaron evidenciados como estrategia de uso regular entre los mismos. De igual manera, la Praxis tutorial requiere de diferentes modos de pensamiento para el abordaje científico en el desarrollo escritural y lingüístico. Los resultados evidencia la importancia que tiene el manejo afectivo emocional en el compromiso que tanto el tutor como el tutoriado deben establecer, ya que esto es lo que permite que fluya la dinámica intelectual y se obtenga el desarrollo exitoso de la gestión tutorial

Palabras Claves: dinámica intelectual; praxis tutorial; inteligencia emocional; postgrado

Abstract: Post-graduate studies have been increasing in recent years, both in quantity offered and in the number of participants enrolled in them, however the number of graduates do not correspond to the number of enrolled. Many culminate their academic charges, but do not fulfill with the presentation of the Title Work requirement for obtaining the nomination, so the intellectual production still does not respond to the expectations that the market and society require. Up to now the different investigations in this regard try to focus the problem towards very specific areas, however the thought processes or the intellectual dynamics that come into play do not seem to have aroused concerns for their investigation, hence the present investigation aimed to identify 
those processes that are implicit during the development of the tutorial praxis, for it, it was framed within a mixed approach, where the quantitative was framed in a descriptive type research, with no experimental design. The population was conformed by 18 teachers with function as a tutor. The information was obtained through a Lickert-type survey. The qualitative approach allowed the researchers a direct approach with their object of study that is Intellectual Dynamics. The information was obtained through interviews with key informants. The use of different strategies: summaries, underlining, questioning, among others were evidenced as a strategy of regular use among them. In the same way, the tutorial Praxis requires different ways of thinking for the scientific approach in the scriptural and linguistic development. The results show the importance of affective emotional management in the commitment that both the tutor and the tutee must establish, since this is what allows the intellectual dynamics to flow and the successful development of the tutorial management

Key Words: intellectual dynamics; praxis tutorial; emotional intelligence; postgraduate

\section{Introducción}

La misión de los estudios de postgrado es formar recursos humanos del más alto nivel, producir conocimiento científico y tecnológico, responder a la demanda de formación de profesionales elevando su formación académica y desempeño profesional, así como contribuir con el desarrollo social, cultural y económico del país. A tal efecto, la Educación Superior a nivel de los Postgrados en general, enfrenta y vive el dilema de la globalización y los diferentes cambios que a nivel mundial se suceden día a día. En este orden de ideas la educación superior y específicamente los postgrados deben atender a la exigente misión que tienen de producir conocimiento formando profesionales altamente calificados y especializados para responder, con su acción profesional transformadora, a las demandas de la realidad social en cuanto al campo profesional especializado, así como a la producción de conocimiento científico y tecnológico.

Sin embargo a lo largo de los años esta misión se ha visto mermada por la baja producción intelectual, es decir el formar profesionales altamente competentes responsables de dar respuesta a una sociedad de manera creativa a través de trabajos de investigación que respondan a las diferentes requerimientos de la sociedad no se está cumpliendo, por lo tanto este sistema educativo ha ido perdiendo efectividad.

En este sentido, los estudios de postgrado representan la educación sistemática de mayor nivel y prestigio de una sociedad en tiempos de globalidad y transformación tecnocientífica mundial. Ante esta realidad, los estudios de postgrado en general deben dirigir su atención y praxis hacia la formación de profesionales con énfasis en la investigación y desarrollo intelectual con criterios de calidad académica, en la cual los docentes del sistema de educación superior no deben ser solamente transmisores sino también creadores de conocimientos.

A este particular, algunas investigaciones pretenden dar respuesta al bajo número de egresados, o por lo menos pretenden explicar las causas de abandono o de deserción en este nivel del sistema educativo. Al respecto, plantea Morles (2004) que los estudios de post grado deberían fundamentarse en la transformación de los procesos culturales y la transmisión del saber, para lograr la máxima potenciación de la producción intelectual encaminada al progreso del país. 
Así se tiene la investigación realizada por Sarmiento En Valarino (2012), acerca del impacto que tienen los trabajos de grado en los estudiantes de postgrado, en el estudio el autor expresa que es casi común en América que los estudiantes se inscriben en los programas de postgrado, y lamentablemente es alto el porcentaje que se ve afectado por lo que el autor considera el síndrome todo menos tesis (TMT). Uno de los aspectos que menciona como importante en las conclusiones de este estudio, lo constituyen causas como las económicas, asimismo, la falta de tiempo o familiares resultaron insignificantes, sin embargo las tutorías resultaron ser un obstáculo de gran peso en estas instituciones, por lo que deficiencias como la falta de tutores, desinterés de algunos, falta de motivación, incompetencia en metodología, entre otras fueron algunas de las causas alegadas. Este mismo artículo complementa que en tal sentido, han venido surgiendo empresas con fines lucrativos que están dando respuesta a los estudiantes al elaborarles las tesis por encargo.

En virtud de lo anteriormente expuesto, esta investigación buscó estudiar los otros factores que también inciden en la producción intelectual, para ello requirió de los docentes a cargo de gestionar, orientar, planificar y ejecutar al lado de sus tutoriados los Trabajos de Grado, su colaboración para indagar el grado de conciencia o conocimiento que poseen sobre su forma de pensar (procesos y eventos cognitivos), los contenidos (estructuras), y su habilidad para organizar y controlar su pensamiento; como procesan y revisan la información, la ajustan o modifican mientras ejercen el rol de tutor, Se trató de ir más allá de los aspectos administrativos involucrados en el desarrollo de los trabajos de investigación; los resultados obtenidos pudieran permitir un mejor aprovechamiento de su rol en pro del beneficio de ambas partes: tutor/tutoriado, lo que igualmente redundaría en la productividad de las instituciones universitarias a nivel de postgrado.

Dentro de este orden de ideas y tomando como base la importancia que tiene para el país los trabajos de investigación como herramienta generadora de nuevas tecnologías y procesos, las investigadoras se formularon las siguientes interrogantes:

¿Qué dinámica intelectual activan en su praxis tutorial los docentes de postgrado?

¿Sobre qué fundamentos teóricos se basa la dinámica intelectual activada durante la praxis tutorial por los docentes de postgrado?

Para dar respuesta a esta formulación del problema se plantean los siguientes objetivos;

\section{Objetivo general}

Analizar la Dinámica Intelectual que Activan en la praxis tutorial, los docentes postgrado.

\section{Objetivos específicos}

- Determinar la Dinámica Intelectual que activan en la praxis tutorial, los docentes de postgrado

- Describir la teoría que fundamenta la Dinámica Intelectual activada durante la praxis tutorial, por los docentes de postgrado

- Describir los elementos que caracterizan la Dinámica Intelectual que activan durante la praxis tutorial, los docentes de postgrado. 
La importancia de la presente investigación radica, en que mediante el análisis de la Dinámica Intelectual que Activan los Docentes de postgrado en su Praxis Tutorial, se pudo obtener una visión sobre los procesos de pensamientos que pone en marcha el tutor durante la tutoría. Esto permitió analizar el proceso educativo durante el desarrollo de la investigación.

Igualmente permitió vincular la permanencia y deserción de los estudiantes de postgrado con la dinámica intelectual puesta en práctica por el docente de la institución, lo que permite corregir posibles desviaciones que se sucedan durante el proceso del diseño del trabajo de investigación. Esto conduce además a disminuir el índice de deserción en los estudiantes del área de postgrado, y a su vez el tutor perfeccione su labor en todos los sentidos. Permitiéndole comparar los objetivos establecidos con los obtenidos.

En este contexto se puede decir que el objetivo principal de esta investigación fue buscar explicaciones relativas a la dinámica intelectual llevada a cabo por los docentes universitarios. El análisis de esta dinámica, permite superar algunas contradicciones existentes entre las exigencias de los alumnos y lo realizado efectivamente por los tutores; todo esto en búsqueda de una mayor fortaleza institucional. Por último, este trabajo sirve de base para una segunda investigación donde se desarrollen las estrategias emocionales, afectivas y cognitivas tanto a nivel de tutores como a nivel del tutoriado; donde también se podrían conocer las competencias investigativas que se requieren en la formación de postgrado. Igualmente representa un aporte a la comunidad científica ya que le suministra aquellos elementos que desarrollen las habilidades y destrezas en los docentes que se inician como tutores.

\section{Metodología}

Para Palella y Martins (2008) "en la investigación, el método implica la elaboración de un plan y la selección de las técnicas más idóneas para su desarrollo" (p.73). La presente investigación tuvo como propósito fundamental analizar la dinámica intelectual que activan en su praxis tutorial los docentes de postgrado; donde hay una realidad que descubrir, construir e interpretar. En las últimas dos décadas diferentes investigadores han propuesto la unión de los procesos cuantitativos y cualitativos vistos como posibles opciones o elecciones de investigación más que como paradigmas, lo que Hernández, Fernández y Baptista (2.008) han denominado "el matrimonio cuantitativo-cualitativo", o "el cruce de enfoques" como lo llaman Lincoln y Gubba citados por los autores ya mencionados. Para los efectos de la presente investigación, las investigadoras asumieron esta propuesta denominada Enfoque Mixto, definido por Teddlie y otros, citado por Hernández y otros. (2.008) como "un proceso que recolecta, analiza y vincula datos cuantitativos y cualitativos en un mismo estudio o una serie de investigaciones para responder a un planteamiento del problema” (p.755).

El Enfoque Mixto presenta diferentes alternativas de combinación, para esta investigación se consideró como el más adecuado el Diseño de dos etapas que implica diferentes niveles de complejidad, para este caso en particular se escogió el de Aplicación Independiente, el cual consiste en aplicar primero un enfoque y luego el otro de manera relativamente independiente dentro de la misma investigación para posteriormente presentar los resultados de manera complementaria.

\section{Enfoque cuantitativo}


En relación al Primer Momento o Enfoque Cuantitativo, esta investigación estuvo enmarcada como una investigación de campo, ya que los datos fueron recogidos directamente de la realidad. (Tamayo y Tamayo, 2007).

En tal sentido, esta investigación fue de tipo descriptivo, Palella y Martins (2008), al respecto afirman "el propósito de este nivel es el de interpretar realidades de hecho. Incluye descripción, registro, análisis e interpretación de la naturaleza actual, composición o procesos de los fenómenos" (p.86).

Dentro de este orden de ideas, se trató de una investigación no experimental ya que en la investigación no hubo manipulación de variables independientes por parte de las investigadoras. Igualmente según Hernández, Fernández y Baptista (2008), definen, “este tipo de investigación como aquella que se realiza sin manipular deliberadamente variables, es decir no se hace variar intencionalmente las variables independientes, lo que se hace es observar fenómenos tal y como se dan en su contexto natural para después analizarlos" (p.36).

Igualmente el diseño es de tipo transeccional, Palella y Martins (2008) al respecto manifiestan "este nivel de investigación se ocupa de recolectar datos en un solo momento y en un tiempo único. Su finalidad es la de describir las variables y analizar su incidencia o interacción en un momento dado, sin manipularlas" (p.88).

\section{Población}

Tamayo y Tamayo (2007) la definen como "la población es la totalidad del fenómeno a estudiar, en donde las unidades de la población tienen una característica común la cual se estudia y da origen a los datos de la investigación" (p.114). Atendiendo a esta definición, la población objeto de esta investigación estuvo constituida por dieciocho (18) docentes que ejercen funciones en Tutoría en postgrado.

\section{Muestra}

En cuanto a la muestra de los Tutores, la misma fue intencional, se utilizó este método ya que estaban accesibles a la investigadora, es decir se tomaron 18 profesores que realizan funciones.

\section{Técnicas e Instrumentos de recolección de datos}

Señala Ruiz (2002) "los instrumentos de medición son procedimientos sistemáticos y estandarizados que permiten observar la conducta humana, a fin de hacer inferencias sobre determinados constructos, rasgos, dimensiones o atributos." (p.23). En tal sentido, se recurrió a la Técnica de la Encuesta y como Instrumento el Cuestionario, al respecto lo define Ruiz (2002) "los cuestionarios, por su parte, son instrumentos conformados por un conjunto de preguntas de naturaleza variada y expresada en diferentes formatos a los fines de sus respuestas." (p.29).

A los efectos de la presente investigación se diseñó un cuestionario conformado por treinta (30) ítems tomando como referencia una escala de estimación tipo Likert con cuatro alternativas de respuestas: 
Siempre (4)

Casi siempre (3)

Casi nunca (2)

Nunca (1)

\section{Validez y confiabilidad}

\section{Validez}

Ruiz (2002), la define como "la exactitud con que pueden hacerse mediciones significativas y adecuadas con un instrumento, en el sentido de que mida realmente el rasgo que pretende medir." (p.73), donde el objetivo del instrumento será probar que los puntajes de la escala realmente permitan distinguir el ejercicio de la acción tutorial. Para ello se recurrió a la Validez de Contenido a través del Juicio de Expertos, donde se tomaron en cuenta a tres especialistas: uno de ellos en el área de cognición y meta cognición, un especialista en diseño de instrumentos y un docente que realiza funciones de tutoría; de la opinión de tales expertos se diseñó el instrumento final.

\section{Confiabilidad}

En lo que se refiere a la confiabilidad señala Hernández y otros (2008), que "es la capacidad que tiene el instrumento de registrar los mismos resultados en repetidas ocasiones, con la misma muestra y bajo las mismas condiciones". (p.41)

\section{Análisis de resultados}

Ese Enfoque de la investigación fue analizada siguiendo los lineamientos de la técnica de la estadística descriptiva, por lo tanto una vez aplicados los cuestionarios se procedió a la tabulación y posterior análisis, para ello los datos fueron condensados en una distribución de frecuencias y análisis porcentual, lo que requirió de la organización, codificación y tabulación de los datos para su análisis respectivo y posterior presentación gráfica que permite una mayor comprensión de los datos obtenidos.

\section{Enfoque cualitativo}

El segundo momento por su parte, estuvo enmarcado dentro del Enfoque Cualitativo, definido por Hernández y otros (2.008):

Puede definirse como un conjunto de prácticas interpretativas que hacen al mundo visible, lo transforman y convierten en una serie de representaciones en forma de observaciones, anotaciones, grabaciones y documentos. Es naturalista (porque estudia a los objetos y seres vivos en sus contextos o ambientes naturales) e interpretativo (pues intenta encontrar sentido a los fenómenos en términos de los significados que las personas le otorguen). (p.9)

En este orden de ideas, este Segundo Momento bajo el Enfoque Cualitativo, le permitió a las investigadoras un acercamiento directo con su objeto de estudio que es la Dinámica Intelectual, vista como un proceso humano que no puede ser objetivizada, es decir los resultados que obtenemos a través del Enfoque Cuantitativo producto del Primer Momento presenta limitaciones para dar respuestas satisfactorias en cuanto al fenómeno de investigación que nos 
ocupa, tal como lo expresa Martínez (2014) “ esta incapacidad hace repercutir su esterilidad y pobreza y frena el avance de los verdaderos conocimientos que necesitamos" (p.21). Igualmente plantea Einstein citado por el mismo autor "en la medida en que las leyes de la matemática se refieren a la realidad, no son ciertas; en la medida en que son ciertas, no se refieren a la realidad" (p.57).

La Dinámica Intelectual vista como una actividad netamente humana solo puede ser analizada y vista bajo la mirada y la narrativa de sus propios protagonistas, el propio " hacedor" de la praxis tutorial es quien nos permite identificar la naturaleza profunda de la realidad a estudiar, de allí tal como indica Martínez (2006) "lo cualitativo (que es el todo integrado) no se opone a lo cuantitativo (que es solo un aspecto), sino que lo implica e integra, específicamente donde sea importante" (p.128). Esta interacción permite el conocimiento como una forma de experiencia que debe ser verificada por métodos integradores, como es el caso de esta investigación. Otro aspecto importante a considerar, es el referido a la credibilidad de los resultados encontrados, visto según Guba (1985), referido por Gutiérrez $(\mathrm{S} / \mathrm{F})$ "a través de las transferibilidad (no generalizables pero si descriptivas o interpretativos de un contexto dado), dependencia (estabilidad de los datos), confirmabilidad (certificación de la existencia de datos para cada interpretación. P.3).

\section{Técnicas e instrumentos}

Este Enfoque de la investigación busca obtener datos de personas y por tratarse de seres humanos lo que interesan son los conceptos, creencias, imágenes mentales, pensamientos, experiencias y vivencias manifestadas en el propio lenguaje de los participantes, cuya finalidad es analizarlos y comprenderlos para luego dar respuestas a las interrogantes de la investigación.

A tal fin, en la Investigación Cualitativa existen diferentes instrumentos, entre ellos la entrevista ya que la misma permite una comunicación entre las partes para la construcción conjunta de significados del tema a investigar. Kvale (1996) mencionado por Martínez (2006) señala "el propósito de la entrevista en Investigación Cualitativa es obtener descripciones del mundo vivido por las personas entrevistadas, con el fin de lograr interpretaciones fidedignas del significado que tienen los fenómenos descritos" (p.140); para este caso en particular la investigadora prefirió el tipo de Entrevista Semi estructurada. En la presente investigación fueron entrevistados tres docentes de postgrado.

Técnicas de Interpretación de la información

Para el procesamiento de la información se utilizó la categorización, la estructuración y la triangulación.

\section{Análisis de los resultados}

Primer momento de la investigación:

En esta primera parte se presenta el análisis de los resultados obtenidos de la aplicación del instrumento a los integrantes de la muestra.

Los mismos están expresados en frecuencias absolutas y porcentuales. De igual manera se hace uso de los gráficos circulares para su mayor comprensión. 
Cuadro $\mathrm{N}^{\mathrm{o}}$ 1:

Distribución de respuestas emitidas por los integrantes de la muestra en relación a la Variable: Dinámica Intelectual. Sub-Dimensión: Tipos de Pensamiento.

\begin{tabular}{|c|c|c|c|c|c|c|c|c|c|}
\hline \multirow{3}{*}{$\begin{array}{l}\text { Variable } \\
\text { Subordinación }\end{array}$} & \multicolumn{9}{|c|}{ Dinámica Intelectual } \\
\hline & \multicolumn{9}{|c|}{ Tipos de Pensamiento } \\
\hline & \multicolumn{2}{|l|}{ Siempre } & \multicolumn{2}{|c|}{ Casi Siempre } & \multicolumn{3}{|c|}{ Casi Nunca } & \multicolumn{2}{|l|}{ Nunca } \\
\hline Items & $\mathrm{F}$ & $\%$ & $\mathrm{~F}$ & $\%$ & $\mathrm{~F}$ & & $\%$ & $\mathrm{~F}$ & $\%$ \\
\hline 3 & 10 & 55,55 & 6 & 33,33 & & 2 & 11,11 & 0 & 0 \\
\hline 4 & 5 & 27,77 & 10 & 55,55 & & 3 & 16,66 & 0 & 0 \\
\hline 5 & 11 & 61,11 & 5 & 27,77 & & 2 & 11,11 & 0 & 0 \\
\hline 7 & 6 & 33,33 & 10 & 55,55 & & 2 & 11,11 & 0 & 0 \\
\hline 12 & 4 & 22,22 & 9 & 50 & & 3 & 16,66 & 2 & 11,11 \\
\hline 13 & 8 & 44,44 & 8 & 44,44 & & 1 & 5,55 & 1 & 5,5 \\
\hline 15 & 12 & 66,66 & 5 & 27,7 & & 1 & 5,55 & 0 & 0 \\
\hline 19 & 5 & 27,77 & 12 & 66,66 & & 1 & 5,55 & 0 & 0 \\
\hline
\end{tabular}

Cuadro $\mathrm{N}^{\mathrm{o}}$ 2:

Distribución de respuestas emitidas por los integrantes de la muestra en relación a la Variable: Dinámica Intelectual. Sub Dimensión: Procesos Mentales

\begin{tabular}{|c|c|c|c|c|c|c|c|c|}
\hline \multirow{3}{*}{$\begin{array}{l}\text { Variable } \\
\text { Subordinación }\end{array}$} & \multirow{3}{*}{\multicolumn{2}{|c|}{$\begin{array}{l}\text { Dinámica Intelectual } \\
\text { Procesos Mentales } \\
\text { Siempre }\end{array}$}} & \multirow{3}{*}{\multicolumn{2}{|c|}{ Casi Siempre }} & \multirow{3}{*}{\multicolumn{2}{|c|}{ Casi Nunca }} & \multirow{3}{*}{\multicolumn{2}{|c|}{ Nunca }} \\
\hline & & & & & & & & \\
\hline & & & & & & & & \\
\hline Ítems & $\mathrm{F}$ & $\%$ & $\mathrm{~F}$ & $\%$ & $\mathrm{~F}$ & $\%$ & $\mathrm{~F}$ & $\%$ \\
\hline 1 & 12 & 66,66 & 5 & 27,77 & 1 & 5,55 & 0 & 0 \\
\hline 2 & 12 & 66,66 & 5 & 27,77 & 1 & 5,55 & 0 & 0 \\
\hline 9 & 5 & 27,77 & 9 & 50 & 3 & 16,66 & 1 & 5,55 \\
\hline 16 & 14 & 77,77 & 4 & 22,22 & 0 & 0 & 0 & 0 \\
\hline 17 & 11 & 61,11 & 6 & 33,33 & 1 & 5,55 & 0 & 0 \\
\hline 18 & 11 & 61,11 & 7 & 38,88 & 0 & 0 & 0 & 0 \\
\hline 20 & 13 & 72,22 & 4 & 22,22 & 1 & 5,55 & 0 & 0 \\
\hline 21 & 11 & 61,11 & 4 & 22,22 & 3 & 16,66 & 0 & 0 \\
\hline 22 & 13 & 72,22 & 5 & 27,77 & 0 & 0 & 0 & 0 \\
\hline 24 & 6 & 33,33 & 11 & 61,11 & 1 & 5,55 & 0 & 0 \\
\hline 25 & 11 & 61,11 & 6 & 33,33 & 1 & 5,55 & 0 & 0 \\
\hline 29 & 15 & 83,33 & 3 & 16,66 & 0 & 0 & 0 & 0 \\
\hline
\end{tabular}

Cuadro $N^{\circ}$ 3:

Distribución de respuestas emitidas por los integrantes de la muestra en relación a la Variable. Dinámica Intelectual. Sub Dimensión: Estrategias Cognitivas y Metacognitivas.

Variable Dinámica Intelectual

Subordinación Estrategias Cognitivas y Metacognitivas 


\begin{tabular}{|c|c|c|c|c|c|c|c|c|c|}
\hline & & Siempre & & Cas & Siempre & Casi Nunca & & Nunca & \\
\hline Ítems & & $\bar{F}$ & $\%$ & $F$ & $\%$ & $F$ & $\%$ & $F$ & $\%$ \\
\hline & 6 & ( & 33,33 & 9 & 50 & 3 & 16,66 & 0 & 0 \\
\hline & 8 & ( & 0 & 9 & 50 & 6 & 33,33 & 3 & 16,66 \\
\hline & 10 & 1 & 55,55 & 3 & 16,66 & 3 & 16,66 & 2 & 11,11 \\
\hline & 11 & 2 & 11,11 & 10 & 55,55 & 4 & 22,22 & 2 & 11,11 \\
\hline & 14 & S & 50 & 8 & 44,44 & 1 & 5,55 & 0 & 0 \\
\hline & 23 & 12 & 66,66 & 6 & 33,33 & 0 & 0 & 0 & 0 \\
\hline & 26 & 12 & 66,66 & 5 & 27,77 & 1 & 5,55 & 0 & 0 \\
\hline & 27 & 1 & 77,77 & 4 & 22,22 & 0 & 0 & 0 & 0 \\
\hline & 28 & 1 & 66,66 & 6 & 33,33 & 0 & 0 & 0 & 0 \\
\hline & 30 & 1 & 83,33 & 3 & 16,66 & 0 & 0 & 0 & 0 \\
\hline
\end{tabular}

\section{Discusión de los resultados}

La dinámica intelectual desarrollada en el transcurso de esta experiencia investigativa, direcciona a que las autoras establezcan un marco de reflexiones producto del devenir de este proceso. A tal efecto, a continuación se presentan a manera de conclusiones, un cuadro de aspectos resaltantes y significantes que constituyen un referente de los hallazgos encontrados en la actividad investigativa finalizada.

1. Se determinó que la Dinámica Intelectual no es un fenómeno aislado dentro de la praxis tutorial, ya que esta viene acompañada de un componente afectivo y experiencial que es lo que hace que la misma sea exitosa.

2. La investigación fue arrojando datos en los que se confirmaban los procedimientos mentales de adquisición, codificación y recuperación de la información por parte de los tutores, por consiguiente estaban implícitas sus competencias investigativas. El uso de diferentes estrategias: resúmenes, subrayado, cuestionamientos, entre otros quedaron evidenciados como estrategia de uso regular entre los mismos. La Praxis tutorial requiere de diferentes modos de pensamiento para el abordaje científico en el desarrollo escritural y lingüístico, lo cual también quedó evidenciado. Todo ello llevó a las investigadoras a buscar el respaldo que en cuanto a teoría pudo dar explicación a otros procesos que se van estableciendo en la praxis tutorial y que es a través de la inteligencia emocional que la dinámica intelectual fluye, logrando potenciar las competencias comunicacionales entre tutor y tutoriado, entre ellas el raport, la empatía, el respeto y el intercambio de ideas. Todo esto es lo que ha permitido ir entendiendo la Dinámica Intelectual.

3. El análisis de los elementos que caracterizan la Dinámica Intelectual de los docentes de postgrado, llevó a concluir que éstos poseen el perfil que les permite desarrollar las competencias investigativas que se requieren como tutor de los trabajos especiales de grado en postgrado, tales como cognitivas, afectivas, comunicacionales, metodológicas, técnicas, procedimentales y estéticas. Pero más allá de ello desarrollar la habilidad para supervisar y entender las propias emociones y la de los tutoriados, discriminar entre ellas, ser capaz de comprenderlos para generar un trabajo cooperativo que le permita entender que lo académico va fluyendo en la medida en que se van generando capacidades sociales y emocionales para el éxito en la vida y por consiguiente en la gestión tutorial. 
4. Los datos extraídos de la encuesta aplicada a los tutores indican que en cuanto a la sub dimensión Tipos de Pensamiento el deductivo, inductivo, analítico, crítico, interrogativo, sistémico y de síntesis representan estrategias de uso común entre los tutores, por lo que van recurriendo a las mismas según las necesidades que se vayan detectando durante el intercambio con el tutoriado, puesto que las diferencias individuales de cada tutor y la de cada tutoriado van direccionando el abordaje más adecuada para el entendimiento investigativo entre ambos.

5. En lo que respecta a la sub dimensión Procesos Mentales, los resultados evidencian como el análisis de lecturas representa una actividad permanente y relevante a la cual tutor recurre como estrategia para la adquisición y codificación de nueva información. De igual forma la realimentación de su gestión como tutor le permite autoevaluarse, corregir fallas y replantearse nuevas estrategias en la planificación en su quehacer tutorial.

6. Para la sub dimensión Estrategias Cognitivas y Metacognitivas se pudo obtener como resultado, que actividades como la posibilidad de fomentar en los tutoriados la visualización de posibles escenarios investigativos, la revisión continua del material escrito, el potenciar en los tutoriados la asociación de los conocimientos con la realidad y la transferencia de las mismas a situaciones futuras y el relacionar conocimientos previos con los actuales, son los que le permiten al tutor reconocer el éxito de su gestión en función de los logros alcanzados por su tutoriado.

7. Del conversatorio con la praxis tutorial representada en esta investigación con la imagen del Búho como fiel exponente de la ciencia, el conocimiento y la sabiduría, quien generó las respuestas contundentes que a cada pregunta proporcionaron los informantes claves; con lo que se puede concluir que es un proceso interactivo, donde hay que establecer un raport, llegar a acuerdos, establecer compromisos, que debe ser de motivación permanente, donde debe haber un conocimiento que medie esta relación e intercambio, donde se propicie un clima armónico, agradable, de respeto, colaboración y compromiso entre las partes, el tutor debe ser flexible, amplio de pensamiento para potenciar saberes, donde el tutor debe conocer sus propias emociones para controlarlas y poder usarlas como herramienta efectiva y exitosa en su gestión. Es lo que en resumidas cuentas permite el cierre exitoso de la gestión tutorial, donde lo anteriormente descrito en este párrafo da explicación de la dinámica intelectual objeto de la presente investigación.

8. Por último, la fundamentación teórica, la experiencia y las vivencias obtenidas durante el desarrollo de la presente investigación, permiten describir la Dinámica Intelectual como la conjunción de estrategias endógenas, estrategias de aprendizaje y procesos cognitivos de orden superior que el individuo activa y pone en práctica ante una situación que amerite aprender o compartir un conocimiento. En el caso del trabajo especial de grado, es toda la conjunción de elementos que interactúan entre sí dentro de lo que es el cerebro, la mente, el pensar de un ser humano para plasmar y llevar a feliz término la meta establecida que es en este caso la culminación exitosa del trabajo especial de grado.

\section{Un conversatorio con la praxis}

Esta segunda parte o segundo momento del análisis de los datos cualitativos, la iniciamos con la conversación directa con la praxis a través de los informantes clave, cuyas identidades ya fueron expuestas; ellos son La Academia, La Universidad y La Cátedra, las siguientes tres preguntas les permitió a cada uno de los informantes narrar sus vivencias en un dialogo ameno, cordial y espontáneo: ¿Cómo es una sesión de tutoría entre usted y su tutoriado?, ¿Cuáles 
considera usted son los aspectos esenciales que debe tener en cuenta un tutor durante la relación tutorial? y por último Desde el punto de Intelectual ¿Cuáles son las fortalezas que debe tener un tutor?.

Cada una de las entrevistas fue grabada y posteriormente transcrita; el contenido de las mismas aparece en el Registro de Entrevista, estas entrevistas arrojaron una masa considerable de información muy difícil de comparar o analizar, por cuanto hubo que realizar una labor para reducir los datos mediante resumen o simplificación de los mismos, buscando así aquella información que mayor relación tuviese o la que mejor ayudaría a describir estructuras significativas que dieran razón de la conducta de los informantes objeto de este estudio con respecto a cada una de las interrogantes, esta simplificación está reflejada en las Estructuras Individuales de cada informante clave. De este proceso se derivó La Estructura General, que no es otra cosa que los argumentos sintetizados visualizados en forma de mapa mental dentro del cual se exponen los datos relevantes que los informantes clave suministraron; donde la investigadora se valió de la imagen del Búho como representante de la sabiduría, la ciencia y el conocimiento para dar respuesta a cada una de las interrogantes. Todo este valioso material informativo, vivencial y práctico permitió a las investigadoras tratar de identificar la naturaleza profunda de la realidad que nos ocupa, además de la estructura dinámica que da razón plena del comportamiento y las manifestaciones de los informantes claves, las cuales estas expresadas en la Síntesis Conceptual a través de la cual se trata de explicar la dinámica intelectual que subyace en la praxis tutorial.

\section{Bibliografía}

Arias, F. (1999). El Proyecto de Investigación. Una Guía para su Elaboración. (2a. Ed.). Caracas: Episteme

Balestrini, M. (1997). Como Elaborar un Proyecto de Investigación. Caracas: Consultores asociados

Barrios, N. (1996). Perfil de un Proceso Tutorial creativo de Trabajo de Grado, enmarcado en el enfoque de interaccionismo Multifactorial dirigido a la Universidad Nacional Experimental Rómulo Gallegos de San Juan de los Morros - Edo. Guárico. (Tesis de Maestría). Universidad Pedagógica Experimental Libertador. Instituto Pedagógico Rafael Alberto Escobar Lara. Maracay.

Calvo, F. (2001). Estadística Aplicada. Barcelona: Deudos

Canales, E (S/F). El Perfil del Tutor Académico. (Documento en línea). Recuperado de http://papyt.xa,vam.mx/media/bhem/dues/pdf/116pdf

Fernández, S. y Cols (1997) Cultura, Inteligencia emocional percibida y ajuste emocional. Un estudio preliminar. Revista electrónica de motivación y emoción. 4, 8-9. Recuperado de http://reme.uji.es/articulos/afernp9912112101/texto.html.

Fuenmayor, F. (2007) Procesos Cognitivos y Metacognitivos Activados por Estudiantes de Educación Media y Profesional cuando resuelven problemas matemáticos. (Trabajo de Maestría). Universidad Pedagógica Experimental Libertador. Instituto Pedagógico Rafael Alberto Escobar Lara. Maracay. 
Gutiérrez, L. (S/F). Paradigmas Cuantitativo y Cualitativa en la Investigación Socioeducativa. Proyecciones y reflexiones. Revista Paradigma. Recuperado de http://www.revistaparadigma.org.ve/Doc./Paradigma96/doc/htm.

Hernández, R., Fernández, C. y Baptista, P. (2008). Metodología de la Investigación. (4a. Ed.). Ciudad de México: McGraw-Hill.

Hernández, J. (2004). El papel del liderazgo del profesor y el tutor en la gestión del proceso educativo. (Documento en línea). Recuperado de http://www.monografias.com/trabajos40/liderazgo-del-profesor/liderazgo

Hurtado, J. (2005). Como formular objetivos de Investigación. Venezuela: Fundación Sypal.

Hurtado, D. (2008) La Configuración: Un recurso para comprender los entramados de las significaciones imaginarias. (Documento en línea). Recuperado de http://www.unizales.edu.co/revistacinde/index.htlm

Martínez, M. (2014). Comportamiento Humano. (2ª Ed.) México: Trillas

Martínez, M (2006). La Investigación Cualitativa (Síntesis Conceptual). (Documento en línea). Recuperado de http://sisbib.unmsm.edu.pe/Brevistas/Investigacion_Psicologia/v09_n1/pdf/a09v9n1.p df

Mateo M. (2001). Metacognición y Educación. Buenos Aires: Aique.

Morles, V. (2004). La educación de postgrado en Venezuela. Panorama y perspectiva. IESALC UNESCO. Caracas: Monte de Ávila

Neisser, U. (1981). Procesos Cognitivos y Realidad. Madrid: Marova

Poggioli, L (S/F). Aprender a enseñar y aprender a aprender. (Documento en línea). Recuperado de http://www.fpolar.org.ve/poggioli/poggprof/.htm

Palella y Martins (2008). Metodología de la Investigación Cuantitativa. Venezuela: Fondoupel

Ruiz, C. (2002). Instrumentos de Investigación Educativa. Venezuela: Cideg

Ruiz, J. (1998). Dirección de los procesos educativos. .Universidad de Pinar del Río. (Documento en línea). Recuperado de http://www.monografias.com/trabajos40/liderazgo-del profesor/liderazgo-delprofesor2.shtml

Tamayo y Tamayo (2007). El Proceso de la Investigación Científica. (6a ed). México, DF. Limusa.

Valarino, E. (2012). Tesis a Tiempo. Barcelona, España: Grupo E, Capitulo 4: El Director es muy Importante. Pp. 129-132. 